\title{
Brain Functional Differences in Drug-Naive Major Depression with Anxiety Patients of Different Traditional Chinese Medicine Syndrome Patterns: A Resting-State fMRI Study
}

\author{
Yi Du, ${ }^{1}$ Jingjie Zhao, ${ }^{1}$ Yongzhi Wang, ${ }^{1}$ Yu Han, ${ }^{2}$ Ligang Deng, ${ }^{3}$ Hongxiao Jia, ${ }^{4}$ \\ Yuan Zhou, ${ }^{5,6}$ Joyce Su, ${ }^{7}$ and $\mathrm{Li} \mathrm{Li} \oplus^{1}$ \\ ${ }^{1}$ Department of Traditional Chinese Medicine, Beijing Friendship Hospital, Capital Medical University, Beijing, China \\ ${ }^{2}$ Your Health \& Wellness International Medical Clinic, Beijing, China \\ ${ }^{3}$ Department of Radiology, Beijing Friendship Hospital, Capital Medical University, Beijing, China \\ ${ }^{4}$ Department of Psychiatry, Beijing Anding Hospital, Capital Medical University, Beijing, China \\ ${ }^{5}$ Key Laboratory of Behavioral Science, Institute of Psychology, Chinese Academy of Sciences, Beijing, China \\ ${ }^{6}$ Department of Psychology, University of Chinese Academy of Sciences, Beijing, China \\ ${ }^{7}$ Department of Biochemistry, Case Western Reserve University, Cleveland, OH, USA
}

Correspondence should be addressed to Li Li; lili@ccmu.edu.cn

Received 18 July 2019; Revised 29 December 2019; Accepted 16 January 2020; Published 18 February 2020

Academic Editor: Johannes Fleckenstein

Copyright (C) $2020 \mathrm{Yi} \mathrm{Du}$ et al. This is an open access article distributed under the Creative Commons Attribution License, which permits unrestricted use, distribution, and reproduction in any medium, provided the original work is properly cited.

\begin{abstract}
Major depressive disorder (MDD), especially combined with anxiety, has a high incidence and low detection rate in China. Literature has shown that patients under major depression with anxiety (MDA) are more likely to nominate a somatic, rather than psychological, symptom as their presenting complaint. In the theory of Traditional Chinese Medicine (TCM), clinical symptoms of MDD patients are mainly categorized into two different syndrome patterns: Deficiency and Excess. We intend to use restingstate functional magnetic resonance imaging (rs-fMRI) to investigate their brain functional differences and hopefully to find their brain function mechanism. For our research, 42 drug-naive MDA patients were divided into two groups ( 21 for Deficiency and 21 for Excess), with an additional 19 unaffected participants in the normal control (NC) group. We took Hamilton Depression Rating Scale (HAMD), Hamilton Anxiety Scale (HAMA), and brain fMRI scan for each group and analyzed the data. We first used Degree Centrality (DC) to map the functional differences in brain regions, utilized these regions as seed points, and used a seedbased functional connectivity (FC) analysis to identify the specific functional connection between groups. The Deficiency group was found to have higher HAMD scores, HAMA scores, and HAMD somatic factor than the Excess group. In the DC analysis, significant decreases were found in the right precuneus of both the Deficiency and Excess groups compared to the NC group. In the FC analysis, the right precuneus showed significant decreased network connectivity with the bilateral cuneus, as well as the right lingual gyrus in the Deficiency group when compared to the NC group and the Excess group. Through our research, it was found that precuneus dysfunction may have a relationship with MDA and Deficiency patients have more severe physical and emotional symptoms, and we realized that a larger sample size and multiple brain mode observations were needed in further research.
\end{abstract}

\section{Introduction}

Major depressive disorder (MDD) is a mental disorder characterized by low emotion, slow thinking, and reduced speech action. It is a leading cause of global burden $(10.3 \%$ of the years of life lived with a physical or mental disability), and the prevalence of MDD is $4.45 \%$ in the United States and $3.02 \%$ in China [1]. MDD patients in China, especially women [2], have been found to complain of somatic symptoms rather than mental symptoms [3]. 60\% of Malaysian Chinese depressive patients complained of somatic symptoms, while only $13 \%$ of Australian depressive 
patients did so [4]. This phenomenon may be related to the traditional Chinese viewpoint towards depression, resulting in greater difficulty in identifying and treating MDD patients. As the prevalence rate, recurrence rate, and disability rate of MDD were high [5], major depression with anxiety (MDA) has been confirmed to have more severe physical symptoms and more suicidal thoughts and behavior and are more difficult to be treated [6].

Chinese MDD patients always complain about somatic symptoms, such as stomach pain, arthralgia, or headache $[1,7]$, and these multiple physical symptoms usually coexist. Traditional Chinese Medicine (TCM) is used to summarize the complex and diverse clinical somatic symptoms by different syndrome patterns and give appropriate treatment. Referring to the internal medicine [8] and clinical study of TCM [9], we chose to research the most important and representative patterns of TCM: Deficiency and Excess. Most somatic symptoms of MDA patients can be summarized by these two patterns and can be easily distinguished (Table 1).

It is difficult to distinguish depression and anxiety and their severity in outpatient quickly, especially in the case of complicated somatization symptoms. Severe MDA patients may have severe impairments, decreased working abilities, and increased risk of incidence rate of comorbid illnesses and may need increased medical treatment [10]. The severity of depression is often determined by psychological scales such as the Hamilton Depression Rating scale (HAMD) [11], 9-item Patient Health Questionnaire (PHQ-9) [12], and Hamilton Anxiety Scale (HAMA) [13]. However, research has showed that such a scale is only effective for short-time illnesses and cannot reflect depression as a chronic illness [14]. Quickly and accurately identifying the severity of depression as a chronic illness is essential for follow-up precise treatment. Our research focused on the brain function mechanism differences between TCM patterns in MDA patients and provided theoretical support for syndrome treatment of TCM.

Resting-state functional magnetic resonance imaging (rs-fMRI) is a noninvasive way to observe brain function and is thus widely used by researchers of brain and mood disorders, including depression [15]. The study of MDD patient brain function showed that there are regions, connections, and network differences from those without MDD. The brain regions which are related to depression are located in the cingulate gyrus [16, 17], prefrontal cortex, cuneus $[18,19]$, insula [20], and gyrus lingualis [21], as well as the default-mode network (DMN) in brain-resting networks [22]. The amygdala [23] and hippocampus [24] were also areas of interest. These regions and networks are related to cognition, memory, and emotion and confirmed the brain function mechanism of depressive patients [25]. Compared to MDD, there were also several functional abnormal regions in MDA patients such as ventral anterior cingulate and amygdala [26], middle temporal gyrus and cuneus [27], and even functional connection abnormal in DMN [28]. Although many functional abnormal brain regions were found in both MDD and MDA, they were relatively concentrated and mostly related to emotional and cognitive function.
Therefore, our hypothesis is that there are even brain functional differences between Deficiency and Excess patterns of MDA patients in these brain regions, and these differences result in different somatic symptoms and TCM patterns. So we intent to use rs-fMRI to research the brain function differences between Deficiency and Excess MDA patients to describe brain function mechanisms of these two TCM patterns and to provide clinical materials to support follow-up TCM syndrome differentiation and treatment of MDA.

\section{Materials and Methods}

2.1. Subjects. The MDA patients were screened from the clinics of Beijing Friendship Hospital and Beijing Anding Hospital. Normal controls were recruited from the local community through advertisements. We screened 42 patients that met the two TCM patterns from 63 first-episode treatment-naive MDA patients (21 Deficiency patients and 21 Excess patients, defined according to TCM syndrome patterns) and recruited 19 matched normal controls (NC). All patient-involved activities were preapproved by the Medical Research Ethics Committee of Beijing Friendship Hospital, Capital Medical University. Written informed consent was obtained from each participant.

For all participants, the inclusion criteria were as follows: (1) between the age of 18 and 65 years; (2) righthanded; and (3) have a Mini-Mental State Examination (MMSE) score $>24[29,30]$. Participants were excluded if they had (1) primary neurological illness, including dementia or stroke; (2) any brain white matter changes in T2weighted magnetic resonance images, including infarction or other vascular lesions and gray matter atrophy; (3) history of any other major psychiatric diseases, such as bipolar disorder, schizophrenia, personality disorder, intellectual disability, and claustrophobia; (4) presence of a medical illness that could impair cognitive functions, such as diabetes; (5) alcohol/drug abuse or dependence; and (6) metallic foreign bodies such as pacemakers, metallic dentures, or amalgam fillings. In addition to complying with the aforementioned general criteria, MDA subjects were subjected to the following additional inclusion criteria: (1) diagnoses by way of structured clinical interviews by two well-trained senior psychiatrists in accordance with the DSM-IV criteria for depressive disorder with anxious distress [31]; (2) 24-item HAMD scores $\geq 18$; (3) Hamilton Anxiety Scale (HAMA) score $\geq 14$; (4) conform to the diagnostic criteria of Deficiency and Excess of TCM syndrome differentiation standard [8]; and (5) medication-free for at least 2 weeks. The additional criteria for normal controls were as follows: (1) HAMD score $<8$ and (2) HAMA score $<7$.

2.2. MRI Data Acquisition. Images were acquired with a 3.0 Tesla GE MRI scanner (SIGNA EXCITE) from the Radiology Department of Beijing Friendship Hospital. The subjects were told to keep their eyes closed and minds relaxed during the scanning and to not fall asleep. 
TABLE 1: Syndrome characteristics of the two TCM syndromes.

\begin{tabular}{lcc}
\hline TCM pattern & Syndrome characteristic & Tongue image and pulse \\
\hline Deficiency & $\begin{array}{c}\text { Thoughtful, suspicious, dizzy, timid, heart palpitations, } \\
\text { insomnia, forgetfulness, loss of appetite, pale complexion }\end{array}$ & Pale tongue, thin white fur, weak pulse \\
\hline Excess & $\begin{array}{c}\text { Depression, impatience, chest swell, flank rib pain, } \\
\text { suffocating, inappetence, stool irregularities }\end{array}$ & Thin and greasy fur, string pulse \\
\hline
\end{tabular}

Whole-brain functional scans were collected in 34 axial slices using an echo-planar matrix $=64 \times 64$, field of view $=220 \times 220 \mathrm{~mm}^{2}$, slice thickness $=4 \mathrm{~mm}$, and slice gap $=0.5 \mathrm{~mm}$. Each functional run contained 240 volumes.

2.3. Imaging Data Preprocessing. Unless otherwise stated, all preprocessing was performed using the Data Processing Assistant for Resting-State fMRI [32], which is based on the Statistical Parametric Mapping (SPM12) [33] program and the Resting-State fMRI Data Analysis Toolkit [34]. Prior to preprocessing, the first 5 volumes were discarded to allow for signal stabilization. The remaining volumes acquired from each subject were corrected for the differences in slice acquisition times. The resultant images were then realigned to correct for small movements that occurred between scans. The resulting maps were then registered into the Montreal Neurological Institute Atlas space with an EPI template, resampling to $3 \mathrm{~mm}$ isotropic voxels. A $6 \mathrm{~mm}$ full-width half-maximum Gaussian kernel was used for spatially smoothing. Then, several sources of spurious or regionally nonspecific variance were removed from the data by regression of nuisance variables including (1) 24 parameters (including 6 head motion parameters, 6 head motion parameters one time point before, and the 12 corresponding squared items) obtained by rigid body head motion correction, (2) the signal averaged over the whole-brain (global signal), (3) the signal averaged over the lateral ventricles, (4) the signal averaged over a region centered in the deep cerebral white matter, and (5) linear and quadratic trends [35]. Temporal filtering $(0.01-0.1 \mathrm{~Hz})$ of the time series was then performed. The volume-based mean framewise displacement (FD), comparing head position variations between the current and previous volumes, was used to quantify head motion across the volumes for each participant [36].

2.3.1. Degree Centrality. Individual degree centrality (DC) maps were generated in a voxel-wise way within a study mask, which is a predefined gray matter mask including tissue with gray matter probabilities greater than $20 \%$ as previously described [37]. First, the preprocessed functional runs were subjected to voxel-based whole-brain correlation analysis. The time course of each voxel from each participant that was within the gray matter mask was correlated with the time course of every other voxel, which resulted in a correlation matrix. An undirected adjacency matrix was then obtained by thresholding each correlation at $r>0.25$ [37-39]. Then, the DC was computed as the number of significant correlations (binarized) or as the sum of the weights of the significant connections (weighted) for each voxel. Finally, the individual-level voxel-wise DC was converted into a $z$-score map by subtracting the mean DC across the entire brain and dividing by the standard deviation of the whole-brain DC [35, 37].

2.3.2. Seed-Based Functional Connectivity. After identifying the region(s) whose DC showed significant across-group differences, we used a seed-based functional connectivity (FC) analysis to identify the specific functional connectivity contributing to the across-group differences. Specifically, the mean time series of each seed region was acquired by averaging the time series of all of the voxels within that region. And then the correlation coefficients between the averaged time course of the seed region with all other voxels in the brain were computed. Finally, the correlation coefficients were converted into $z$-values using Fisher's r-to-z transformation to improve their normality.

2.4. Statistics. One-way ANOVA analysis was conducted to test whether there were any differences in DC or FC while taking the head motion measured by the mean FD as no interests of covariates.

Standard error method was used for data analysis, and significant group differences were obtained with a clusterwise FWE corrected $P$ value of 0.05 for multiple comparisons (individual voxel threshold, $P<0.001$ ). If the main effect was statistically significant, simple effect analyses were performed for the averaged effect size extracted from the clusters with significant effects using SPSS v19.0.

\section{Results}

3.1. Demographic and Clinical Data. There were no significant differences in age, dender, educational years, and head motion measured by the mean FD and the number of scrubbing of bad time points. However, we do see significant difference in the HAMD score, HAMA score, and HAMD somatic factor between Deficiency and Excess groups (Table 2).

3.2. Degree Centrality. In the NC group, the spatial distribution of the weighted DC was highly localized in the posterior cingulate/ventral precuneus, occipital lobe, middle cingulate cortex (MCC), anterior cingulate cortex/medial prefrontal cortices, lateral prefrontal cortex, inferior parietal regions, insula (Figure 1(a)). Both in the Deficiency and Excess groups, the spatial distribution of the weighted DC was also localized in the abovementioned regions, but the clusters were smaller (Figures 1(b) and 1(c)). Compared to the NC group, significant decreases in the weighted DC were 
TABLe 2: Demographic and clinical characteristics of the participants.

\begin{tabular}{|c|c|c|c|c|}
\hline & $\mathrm{NC}(n=19)$ & Deficiency $(n=21)$ & Excess $(n=21)$ & $P$ value \\
\hline Age & $46.79 \pm 13.97$ & $39.62 \pm 12.39$ & $46.38 \pm 13.28$ & 0.151 \\
\hline Gender $(\mathrm{M} / \mathrm{F})$ & $4 / 15$ & $6 / 15$ & $4 / 17$ & 0.585 \\
\hline Education (years) & $12.52 \pm 3.45$ & $11.90 \pm 2.93$ & $11.28 \pm 2.77$ & 0.444 \\
\hline HAMD score & - & $29.24 \pm 6.65$ & $24.76 \pm 6.54$ & 0.034 \\
\hline HAMA score & - & $26.48 \pm 5.24$ & $19.81 \pm 5.20$ & 0.000 \\
\hline HAMD somatic factor & - & $9.14 \pm 1.49$ & $5.33 \pm 1.31$ & 0.000 \\
\hline Head motion: mean FD & $0.15 \pm 0.04$ & $0.13 \pm 0.07$ & $0.14 \pm 0.04$ & 0.276 \\
\hline
\end{tabular}

HAMD, Hamilton Depression Rating Scale; HAMA, Hamilton Anxiety Rating Scale; NC, normal controls.

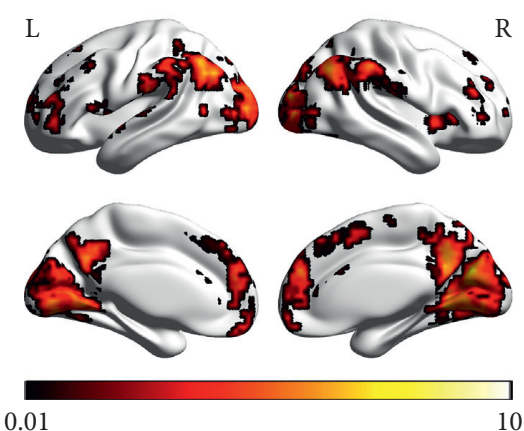

(a)
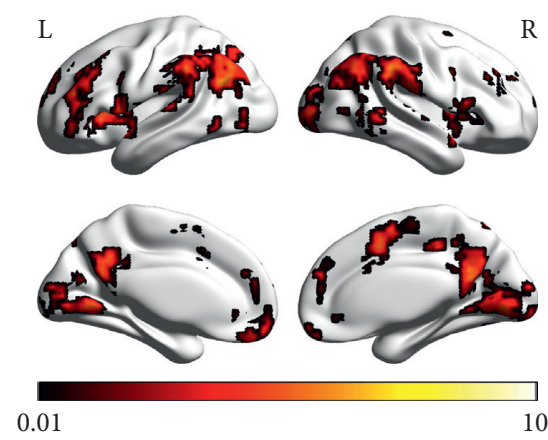

(b)

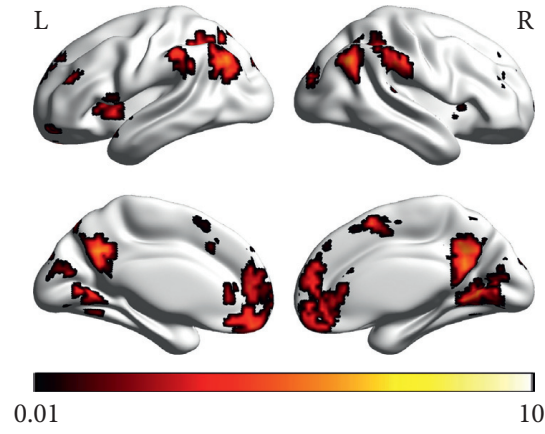

(c)

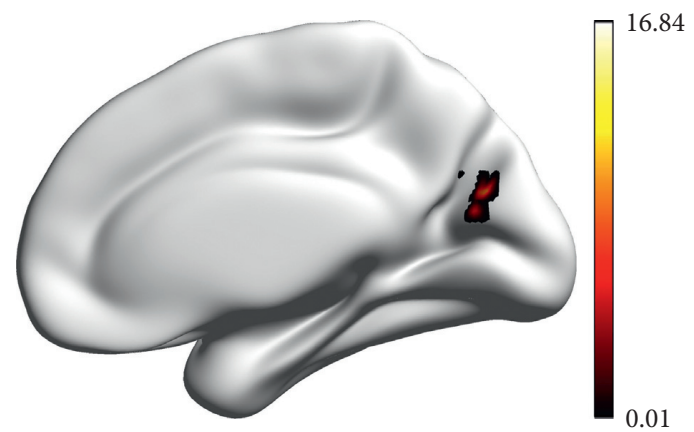

(d)

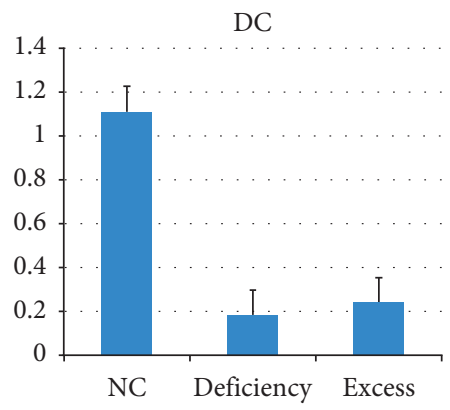

(e)

FIGURE 1: Degree centrality (DC) in each group and differences across groups. (a) DC in the NC group, (b) DC in the Deficiency group, (c) DC in the Excess group, (d) ANOVA results, and (e) Post hoc comparisons. The dark and light colors indicate the $P$ value. The deeper the color, the smaller the $P$ value.

found in the right precuneus in both of the Deficiency group and Excess group (Figures 1(d) and 1(e), Table 3).

3.3. Seed-Based Functional Connectivity. We used the right precuneus which showed significance between group differences as the seed regions for the mapping of the functional connectivity network. In general, the right precuneus showed significant positive connectivity with the regions in a default-mode network (DMN), such as the posterior cingulate cortex and medial prefrontal cortex, and showed significant negative connectivity with the regions in 
TABLE 3: DC differences in the three groups.

\begin{tabular}{lcccccc}
\hline Cluster location & NC & Deficiency & Excess & NC vs. Deficiency & NC vs. Excess & Deficiency vs. Excess \\
\hline Right precuneus & $1.11 \pm 0.51$ & $0.18 \pm 0.52$ & $0.24 \pm 0.51$ & $<0.00$ & $<0.001$ & 0.712 \\
\hline
\end{tabular}

DC, degree centrality; NC, normal controls.

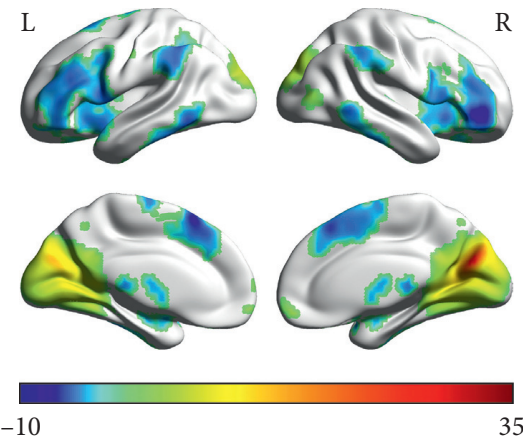

(a)

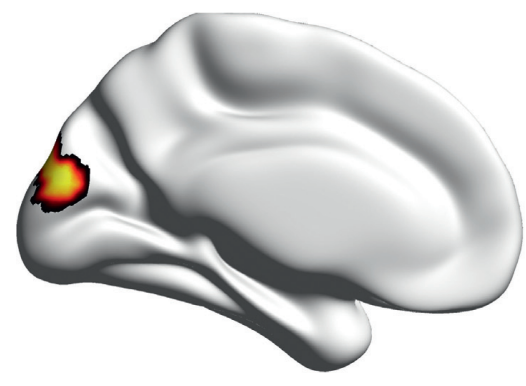

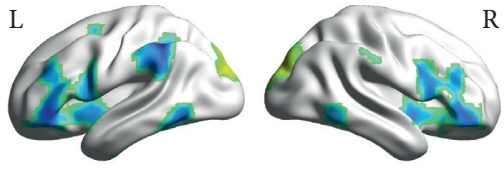

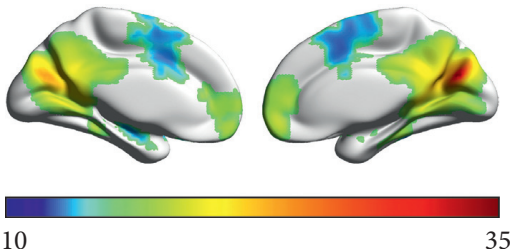

(b)
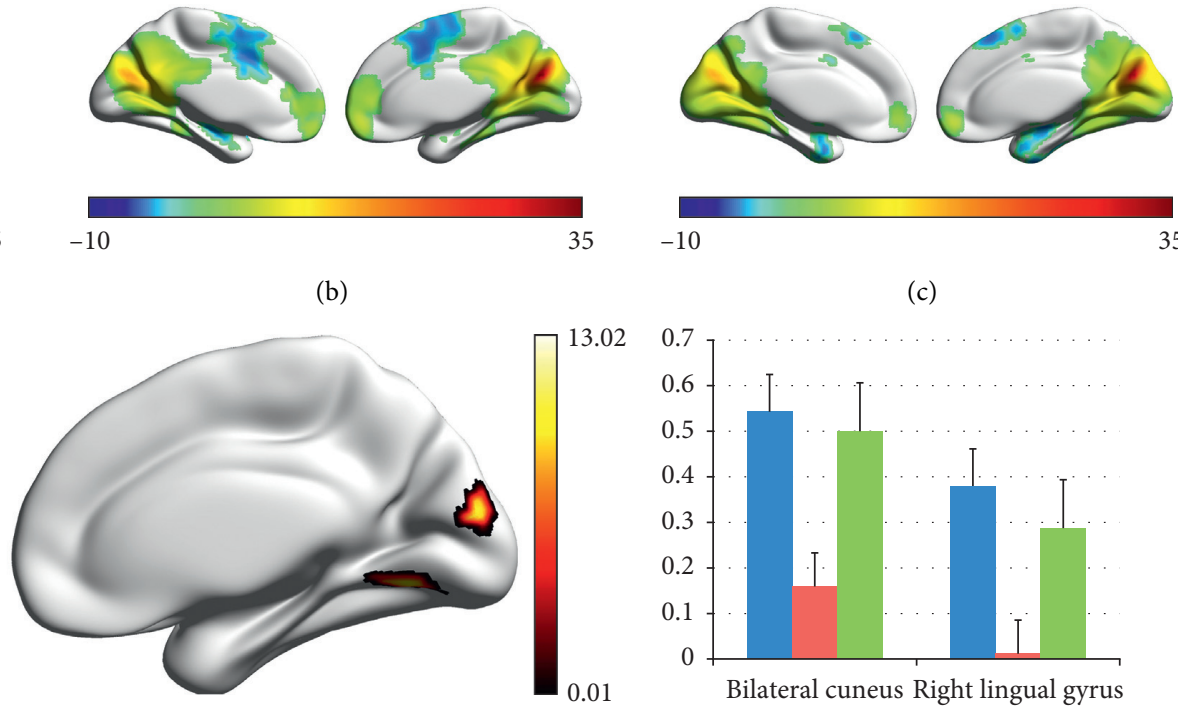

(c)

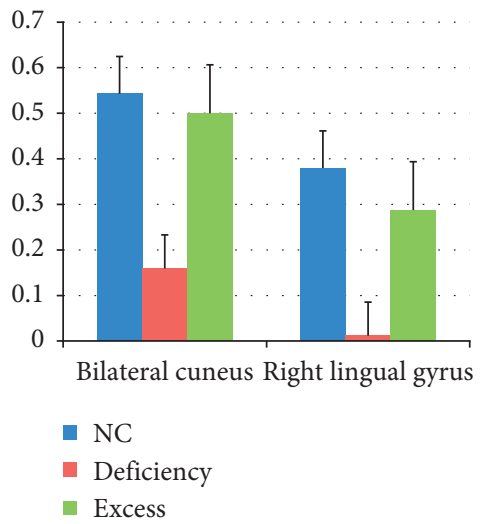

(e)

Figure 2: Functional connectivity (FC) of the right precuneus in each group and differences across groups. (a) DC in the NC group, (b) DC in the Deficiency group, (c) DC in the Excess group, (d) ANOVA results, and (e) post-hoc comparisons. The warm and cold colors in (a-c) indicate the brain regions with significantly increased and decreased FC. The dark and light colors in (d) indicate the $P$ value; the deeper the color, the smaller the $P$ value.

an executive control network (ECN), such as the bilateral prefrontal cortex and parietal cortex, and the regions in a salience network (SAN), such as the bilateral anterior insula and cingulate cortex, in each group (Figures 2(a)-2(c)). ANOVA found significant differences in the connectivity between the right precuneus and the bilateral cuneus as well as the right lingual gyrus (Figure 2(d)). Post-hoc comparisons revealed that the connectivity was decreased in the Deficiency group compared to the NC group and the Excess group, but there were no differences between the Deficiency group and the NC group (Figure 2(e) and Table 4).

\section{Discussion}

TCM has a history of more than 2,000 years. It is a medical theory system that has been gradually formed and developed through long-term medical practice under the guidance of simple materialism and dialectical thinking. Deficiency and
Excess are two most important TCM types with outstanding symptoms in MDD patients $[40,41]$ and usually to be studied as typical syndrome patterns of MDD [42, 43], so we choose them for observation.

According to the scale analysis, we found that the HAMD scores, HAMA scores, and HAMD somatic factor of the Deficiency group were much higher than those of the Excess group. This result had been reported by previous researches $[44,45]$. They suggest that the Deficiency patients had longer disease course and more serious somatic anxiety. Therefore, these two different TCM syndrome patterns can reflect the severity of MDA to a certain extent.

We used the weighted DC to analyze the rs-fMRI data, which demonstrated that all the three groups' spatial distributions of the weighted DC were highly localized in the DMN and dorsal attention network (DAN). These brain networks play a central role in resting-state research $[46,47]$. Then when we used the right precuneus as a seed to calculate 
TABLE 4: FC differences in three groups.

\begin{tabular}{lcccccc}
\hline Cluster location & NC & Deficiency & Excess & NC vs. Deficiency & NC vs. Excess & Deficiency vs. Excess \\
\hline Bilateral cuneus & $0.54 \pm 0.36$ & $0.16 \pm 0.26$ & $0.50 \pm 0.27$ & $<0.001$ & 0.647 & $<0.001$ \\
Right lingual gyrus & $0.38 \pm 0.32$ & $0.01 \pm 0.19$ & $0.29 \pm 0.27$ & $<0.001$ & 0.272 & $<0.001$ \\
\hline
\end{tabular}

FC, functional connectivity; NC, normal controls.

functional connectivity, it showed significantly positive connectivity with the regions in DMN and negative connectivity with the regions in ECN and SAN in general. These distributions are similar to those reported by previous studies [48-50]. It may be indicated that the function of selfconsciousness [51], spontaneous thinking [52], and selfrelated processes [53] were deteriorated in MDA patients.

At the same time, we found that the precuneus was a very important region for MDA patients. Compared with the NC participants, both Deficiency and Excess patients had decreased brain function in the right precuneus. Previous studies have also shown that depressive patients have abnormal brain function in the precuneus $[54,55]$. This region is very important for self-reflection processes and potentially plays a role in mental imagery and episodic/autobiographical memory retrieval [56-58]. The precuneus also assists other brain regions in performing functions such as information processing, especially in regards to emotion regulation $[59,60]$. Furthermore, the precuneus is a major hub of brain organization and a central node of DMN $[61,62]$. It is involved in a variety of information processing states $[63,64]$. There were additional reports that the depressive patients' precuneus made functional connectivity separation of the brain network and that dissociated largescale networks may have contributed to the clinical expression of depression [65]. Although we found that both Deficiency and Excess groups' patients have decreased function in the right precuneus in comparison with the NC group, there were no significant differences between the two TCM syndrome pattern groups. It indicates that the dysfunction of the right precuneus may be the manifestation of brain function abnormality in MDA patients.

From our study, compared with NC and Excess groups, the Deficiency group showed negative connectivity with the bilateral cuneus as well as the right lingual gyrus. There are also abnormal functions of these two brain regions to be found in panic disorder patients [66]. While the cuneus has a function of integrating the somatosensory information with other sensory stimuli and cognitive processes such as attention, learning, and memory [67], the lingual gyrus is a brain region responsible for supporting visual memory [68]. Furthermore, the precuneus was also activated in many version tasks $[69,70]$. Research had also shown that depression and anxiety are primarily correlated with functional deficits in the precuneus-related network [71].

However, we also notice that we need larger samples and even multiple brain mode observations to confirm if the two TCM syndrome patterns have more significant brain regions and network functional differences in precuneus or other brain regions in future research.

\section{Conclusions}

In summary, there are differences in brain function between the two different TCM syndrome patterns of MDA patients. Based on our research, it was found that precuneus dysfunction may have a relationship with MDA and brain functional connectivity differences, and we could find out that the Deficiency patients have more severe physical and emotional symptoms in MDA patients. At the same time, we realized that a larger sample size and multiple brain mode observations were needed in further research.

$\begin{array}{ll}\text { Abbreviation } \\ \text { MDD: } & \text { Major depressive disorder } \\ \text { MDA: } & \text { Major depression with anxiety } \\ \text { TCM: } & \text { Traditional Chinese Medicine } \\ \text { rs- } & \text { Resting-state functional magnetic resonance } \\ \text { fMRI: } & \text { imaging } \\ \text { DC: } & \text { Degree Centrality } \\ \text { FC: } & \text { Functional connectivity } \\ \text { HAMD: } & \text { Hamilton Depression Rating Scale } \\ \text { HAMA: } & \text { Hamilton Anxiety Scale } \\ \text { DMN: } & \text { Default-mode network } \\ \text { ECN: } & \text { Executive control network } \\ \text { SAN: } & \text { Salience network. }\end{array}$

\section{Data Availability}

All data generated or analyzed during this study are included within the article.

\section{Conflicts of Interest}

The authors declare there are no conflicts of interest regarding the publication of this article.

\section{Authors' Contributions}

Yi Du, Han $\mathrm{Yu}$, and Hongxiao Jia contributed by examining the patients and writing the manuscript. Jingjie Zhao, Yongzhi Wang, and Joyce Su contributed to drafting and revising the manuscript. Lili contributed to conception and design of the study. Ligang Deng contributed to make FMRI acquisition. Yuan Zhou contributed to design the FMRI parameter and analysis data. All authors read and approved the final manuscript.

\section{Acknowledgments}

This study was supported by grants from the National Natural Science Foundation of China (grant no. 81673737), 
the Beijing Natural Science Foundation (grant no. 7172063), the Beijing Administration of Traditional Chinese Medicine (grant no. JJ2018-51), the Beijing Municipal Administration of Hospitals Incubating Program (grant code: PZ2017024), and the Beijing Health System Training Program for High Level Technique Talents (grant no. 2014-3-001).

\section{References}

[1] K. Smith, "Mental health: a world of depression," Nature, vol. 515, no. 7526, p. 181, 2014.

[2] C. Yu, J. Lyu, Y. Chen et al., "Epidemiology of major depressive episodes among Chinese adults aged 30-79 years: data from the China kadoorie biobank," Zhonghua Liu Xing Bing Xue Za Zhi, vol. 36, no. 1, pp. 52-56, 2015.

[3] D. S. F. Yu and D. T. F. Lee, "Do medically unexplained somatic symptoms predict depression in older Chinese?" International Journal of Geriatric Psychiatry, vol. 27, no. 2, pp. 119-126, 2012.

[4] G. Parker, Y.-C. Cheah, and K. Roy, "Do the Chinese somatize depression? A cross-cultural study," Social Psychiatry and Psychiatric Epidemiology, vol. 36, no. 6, pp. 287-293, 2001.

[5] M. Y. Zhang, "Depression is a common disease," Chinese Journal of Psychiatry, vol. 44, p. 46, 2011.

[6] D. P. Goldberg, H.-U. Wittchen, P. Zimmermann, H. Pfister, and K. Beesdo-Baum, "Anxious and non-anxious forms of major depression: familial, personality and symptom characteristics," Psychological Medicine, vol. 44, no. 6, pp. 12231234, 2014.

[7] Medinati and Z. Tao, "Physical characteristics of depression in outpatient of general hospital," Chinese Practical Medicine, vol. 7, pp. 88-89, 2012.

[8] Y. Wu and X. Wang, Chinese Medicine Internal Medicine, China Traditional Chinese Medicine Press, Beijing, China, 9th edition, 2012.

[9] H. Macpherson, B. Elliot, A. Hopton, H. Lansdown, and S. Richmond, "Acupuncture for depression: patterns of diagnosis and treatment within a randomised controlled trial," Evidence-based Complementary and Alternative Medicine, vol. 2013, pp. 286048-9, 2013.

[10] R. Saveanu, A. Etkin, A.-M. Duchemin et al., "The international study to predict optimized treatment in depression (iSPOT-D): outcomes from the acute phase of antidepressant treatment," Journal of Psychiatric Research, vol. 61, pp. 1-12, 2015.

[11] J. B. W. Williams, "A structured interview guide for the Hamilton Depression Rating Scale," Archives of General Psychiatry, vol. 45, no. 8, pp. 742-747, 1988.

[12] K. S. R. Kroenke, R. L. Spitzer, and J. B. W. Williams, "The PHQ-9," Journal of General Internal Medicine, vol. 16, no. 9, pp. 606-613, 2001.

[13] W. Maier, R. Buller, M. Philipp, and I. Heuser, "The Hamilton Anxiety Scale: reliability, validity and sensitivity to change in anxiety and depressive disorders," Journal of Affective Disorders, vol. 14, no. 1, pp. 61-68, 1988.

[14] M. Worboys, "The Hamilton Rating Scale for Depression: the making of a "gold standard" and the unmaking of a chronic illness, 1960-1980," Chronic Illness, vol. 9, no. 3, pp. 202-219, 2013.

[15] J. Brakowski, S. Spinelli, N. Dörig et al., "Resting state brain network function in major depression-depression symptomatology, antidepressant treatment effects, future research," Journal of Psychiatric Research, vol. 92, pp. 147-159, 2017.
[16] Z. Yao, L. Wang, Q. Lu, H. Liu, and G. Teng, "Regional homogeneity in depression and its relationship with separate depressive symptom clusters: a resting-state fMRI study," Journal of Affective Disorders, vol. 115, no. 3, pp. 430-438, 2009.

[17] D Li, X. Huang, Q. Wu et al., "Brain functions in major depressive disorder: a resting-state functional magnetic resonance imaging study," Sheng Wu Yi Xue Gong Cheng Xue Za Zhi, vol. 27, pp. 16-19, 2010.

[18] Q.-Z. Wu, D.-M. Li, W.-H. Kuang et al., “Abnormal regional spontaneous neural activity in treatment-refractory depression revealed by resting-state fMRI," Human Brain Mapping, vol. 32, no. 8, pp. 1290-1299, 2011.

[19] F. Liu, W. Guo, L. Liu et al., "Abnormal amplitude lowfrequency oscillations in medication-naive, first-episode patients with major depressive disorder: a resting-state fMRI study," Journal of Affective Disorders, vol. 146, no. 3, pp. 401-406, 2013.

[20] C.-H. Liu, X. Ma, L.-P. Song et al., "Alteration of spontaneous neuronal activity within the salience network in partially remitted depression," Brain Research, vol. 1599, pp. 93-102, 2015.

[21] L. Wang, D. Wenji, Y. Su et al., "Amplitude of low-frequency oscillations in first-episode, treatment-naive patients with major depressive disorder: a resting-state functional MRI study," PLoS. One.vol. 7, no. 10, Article ID e48658, 2012.

[22] L.-L. Zeng, S. Hhen, H. Shen et al., "Identifying major depression using whole-brain functional connectivity: a multivariate pattern analysis," Brain, vol. 135, no. 5, pp. 1498-1507, 2012.

[23] I. M. Veer, C. Beckmann, M. J. van Tol et al., "Whole brain resting-state analysis reveals decreased functional connectivity in major depression," Frontiers in Systems Neuroscience, vol. 4, 2010.

[24] X. Cao, Z. Liu, C. Xu et al., "Disrupted resting-state functional connectivity of the hippocampus in medication-naïve patients with major depressive disorder," Journal of Affective Disorders, vol. 141, no. 2-3, pp. 194-203, 2012.

[25] K. Vassilopoulou, M. Papathanasiou, I. Michopoulos et al., "A magnetic resonance imaging study of hippocampal, amygdala and subgenual prefrontal cortex volumes in major depression subtypes: melancholic versus psychotic depression," Journal of Affective Disorders, vol. 146, no. 2, pp. 197-204, 2013.

[26] A. Etkin and A. F. Schatzberg, "Common abnormalities and disorder-specific compensation during implicit regulation of emotional processing in generalized anxiety and major depressive disorders," American Journal of Psychiatry, vol. 168, no. 9, pp. 968-978, 2011.

[27] C. E. Waugh, J. P. Hamilton, M. C. Chen, J. Joormann, and I. H Gotlib, "Neural temporal dynamics of stress in comorbid major depressive disorder and social anxiety disorder," Biology of Mood \& Anxiety Disorders, vol. 2, no. 1, 2012.

[28] C. Andreescu, M. Wu, M. A. Butters, J. Figurski, C. F. Reynolds, and H. J. Aizenstein, "The default mode network in late-life anxious depression," The American Journal of Geriatric Psychiatry, vol. 19, no. 11, pp. 980-983, 2011.

[29] M. F. Folstein, S. E. Folstein, and P. R. McHugh, "Mini-mental state," Journal of Psychiatric Research, vol. 12, no. 3, pp. 189-198, 1975 .

[30] M. Zhang, Guidelines for the prevention and treatment of dementia in the elderly, Peking University Medical Press, Beijing, China, 2007.

[31] M. B. First, R. L. Spitzer, G. Miriam, and J. B. W. Williams, Structured Clinical Interview for DSM-IV axis I Disorders: 
SCID-I: Clinical Version: Administration Booklet, American Psychiatric Press, Philadelphia, Washington, DC, USA, 1997.

[32] Z. Y. Yan, "DPARSF: a MATLAB toolbox for "pipeline" data analysis of resting-state fMRI," Frontiers in System Neuroscience, vol. 4, no. 13, 2010.

[33] H. Han and J. Park, "Using SPM 12's second-level bayesian inference procedure for fMRI analysis: practical guidelines for end users," Frontiers in Neuroinformatics, vol. 12, no. 1, 2018.

[34] D. Z. Song, X. Y. Long, S.-F. Li et al., "REST: a toolkit for resting-state functional magnetic resonance imaging data processing," PLoS One, vol. 6, Article ID e25031, 2011.

[35] C. G. Yan, R. C. Craddock, and Y. He, "Addressing head motion dependencies for small-world topologies in functional connectomics," Frontiers in Human Neuroscience, vol. 7, p. 910, 2013.

[36] M. Goto, O. Abe, H. Yamasue, T. Gomi, and T. Takeda, "Head motion and correction methods in resting-state functional MRI," Magnetic Resonance in Medical Sciences, vol. 15, no. 2, pp. 178-186, 2016.

[37] X.-N. Zuo, R. Ehmke, M. Mennes et al., "Network centrality in the human functional connectome," Cerebral Cortex, vol. 22, no. 8, pp. 1862-1875, 2012.

[38] R. L. Buckner, J. Sepulcre, T. Talukdar et al., "Cortical hubs revealed by intrinsic functional connectivity: mapping, assessment of stability, and relation to Alzheimer's disease," Journal of Neuroscience, vol. 29, no. 6, pp. 1860-1873, 2009.

[39] C.-G. Yan, B. Cheung, C. Kelly et al., "A comprehensive assessment of regional variation in the impact of head micromovements on functional connectomics," NeuroImage, vol. 76, pp. 183-201, 2013.

[40] L. Xu, Yu Chen, M. Wang, X. Zhou, and S. Liu, "Clinical syndrome differentiation of depression," Journal of Hubei University of Traditional Chinese Medicine, vol. 19, pp. 37-40, 2017.

[41] G. Li, G. Guo, S. Chen, H. Wang, and H. Zhou, "Classification and analysis of TCM syndromes of depression based on literature," Chinese Journal of Traditional Chinese Medicine, vol. 34, pp. 876-879, 2016.

[42] Y. Ma, Z. Chen, W. Jin et al., "Preliminary observation on the treatment of depression by integrated traditional Chinese and western medicine," Clinical Study of Traditional Chinese Medicine, vol. 1, pp. 6-7, 2011.

[43] C. Zhu and Li Xia, "Quzhao. Guipi decoction for depression of deficiency of heart and spleen," Jilin Traditional Chinese Medicine, vol. 34, pp. 695-699, 2014.

[44] C. Li, W. F. Jinqiu, and J. Wu, "Investigation on the relationship between common emotional symptoms and TCM classification of depressive patients in Shenyang," China Clinical Rehabilitation, vol. 47, pp. 42-45, 2006.

[45] C. Chen, Z. Chen, S. Hu, H. Zhang, M. Cao, and Y. Liu, "Factor analysis of Hamilton depression scale of TCM syndrome of depression," Journal of Hunan College of Traditional Chinese Medicine, vol. 04, pp. 32-34, 2003.

[46] M. E. Raichle, “The brain's default mode network," Annual Review of Neuroscience, vol. 38, no. 1, pp. 433-447, 2015.

[47] R. Esposito, F. Cieri, P. Chiacchiaretta et al., "Modifications in resting state functional anticorrelation between default mode network and dorsal attention network: comparison among young adults, healthy elders and mild cognitive impairment patients," Brain Imaging and Behavior, vol. 12, no. 1, pp. 127-141, 2018.

[48] X. Cui, W. Guo, Y. Wang et al., "Aberrant default mode network homogeneity in patients with first-episode treatment-naive melancholic depression," International Journal of Psychophysiology, vol. 112, pp. 46-51, 2017.
[49] S. L. Sommerfeldt, K. R. Cullen, G. Han et al., "Executive attention impairment in adolescents with major depressive disorder," Journal of Clinical Child and Adolescent Psychology, vol. 45, no. 1, pp. 69-83, 2016.

[50] A. Dutta, S. McKie, and J. F. W. Deakin, "Resting state networks in major depressive disorder," Psychiatry Research: Neuroimaging, vol. 224, no. 3, pp. 139-151, 2014.

[51] D. A. Gusnard, E. Akbudak, G. L. Shulman, and M. E. Raichle, "Medial prefrontal cortex and self-referential mental activity: relation to a default mode of brain function," Proceedings of the National Academy of Sciences, vol. 98, no. 7, pp. 42594264, 2001.

[52] M. F. Mason, M. I. Norton, J. D. Van Horn, D. M. Wegner, S. T. Grafton, and C. N. Macrae, "Wandering minds: the default network and stimulus-independent thought," Science, vol. 315, no. 5810, pp. 393-395, 2007.

[53] C. Lemogne, H. Mayberg, L. Bergouignan et al., "Self-referential processing and the prefrontal cortex over the course of depression: a pilot study," Journal of Affective Disorders, vol. 124, no. 1-2, pp. 196-201, 2010.

[54] N. L. Nixon, P. F. Liddle, E. Nixon, G. Worwood, M. Liotti, and L. Palaniyappan, "Biological vulnerability to depression: linked structural and functional brain network findings," British Journal of Psychiatry, vol. 204, no. 4, pp. 283-289, 2014.

[55] M.-J. Van Tol, M. Li, C. D. Metzger et al., "Local cortical thinning links to resting-state disconnectivity in major depressive disorder," Psychological Medicine, vol. 44, no. 10, pp. 2053-2065, 2014.

[56] M. Cabanis, M. Pyka, S. Mehl et al., "The precuneus and the insula in self-attributional processes," Cognitive, Affective, \& Behavioral Neuroscience, vol. 13, no. 2, pp. 330-345, 2013.

[57] A. E. Cavanna, "The precuneus and consciousness," CNS Spectrums, vol. 12, no. 7, pp. 545-552, 2007.

[58] A. E. Cavanna and M. R. Trimble, "The precuneus: a review of its functional anatomy and behavioural correlates," Brain, vol. 129, no. 3, pp. 564-583, 2006.

[59] Y. Chen, Z. Liu, J. Zhang et al., "Precuneus degeneration in nondemented elderly individuals with APOE $\varepsilon 4$ : evidence from structural and functional MRI analyses," Human Brain Mapping, vol. 38, no. 1, pp. 271-282, 2017.

[60] S. J. Ferri and G. Hajcak, "Emotion regulation and amygdalaprecuneus connectivity: focusing on attentional deployment," Cognitive, Affective, \& Behavioral Neuroscience, vol. 16, pp. 1-12, 2016.

[61] E. P. T. Bruner and X. Chen, "Evidence for expansion of the precuneus in human evolution," Brain Structure and Function, vol. 222, no. 2, pp. 1-8, 2017.

[62] P. Fransson and G. Marrelec, "The precuneus/posterior cingulate cortex plays a pivotal role in the default mode network: evidence from a partial correlation network analysis," Neuroimage, vol. 42, no. 3, pp. 1178-1184, 2008.

[63] A. V. Utevsky, D. V. Smith, and S. A. Huettel, "Precuneus is a functional core of the default-mode network," The Journal of Neuroscience, vol. 34, no. 3, pp. 932-940, 2014.

[64] R. Jones and J. Bhattacharya, "A role for the precuneus in thought-action fusion: evidence from participants with significant obsessive-compulsive symptoms," NeuroImage: Clinical, vol. 4, pp. 112-121, 2014.

[65] D. Peng, E. B. Liddle, S. J. Iwabuchi et al., "Dissociated largescale functional connectivity networks of the precuneus in medication-naïve first-episode depression," Psychiatry Research: Neuroimaging, vol. 232, no. 3, pp. 250-256, 2015.

[66] C.-H. Lai and Y.-T. Wu, "Decreased regional homogeneity in lingual gyrus, increased regional homogeneity in cuneus and 
correlations with panic symptom severity of first-episode, medication-naïve and late-onset panic disorder patients," Psychiatry Research: Neuroimaging, vol. 211, no. 2, pp. 127131, 2013.

[67] D. D. Price, "Psychological and neural mechanisms of the affective dimension of pain," Science, vol. 288, pp. 1769-1772, 2000.

[68] J. Bogousslavsky, J. Miklossy, J. P. Deruaz, G. Assal, and F. Regli, "Lingual and fusiform gyri in visual processing: a clinico-pathologic study of superior altitudinal hemianopia," Journal of Neurology, Neurosurgery \& Psychiatry, vol. 50, no. 5, pp. 607-614, 1987.

[69] M. Uchimura, T. Nakano, Y. Morito, H. Ando, and S. Kitazawa, "Automatic representation of a visual stimulus relative to a background in the right precuneus," European Journal of Neuroscience, vol. 42, no. 1, pp. 1651-1659, 2015.

[70] M. Freton, C. Lemogne, L. Bergouignan, P. Delaveau, S. Lehéricy, and P. Fossati, "The eye of the self: precuneus volume and visual perspective during autobiographical memory retrieval," Brain Structure and Function, vol. 219, no. 3, pp. 959-968, 2014.

[71] C. Yuan, H. Zhu, Z. Ren et al., "Precuneus-related regional and network functional deficits in social anxiety disorder: a resting-state functional MRI study," Comprehensive Psychiatry, vol. 82, pp. 22-29, 2017. 